\title{
Representação dos Padrões Atmosféricos Relacionados à Zona de Con- vergência do Atlântico Sul Através de um Modelo Acoplado Atmosfera-Biosfera.
}

\author{
Representation of Atmospheric Patterns Related to the South Atlantic Convergence Zone by \\ an AtmosphereBiosphere Coupled Model.
}

\author{
Leonardo Preza Rodrigues ${ }^{1}$, Mônica Carneiro Alves Senna ${ }^{2} e$ Marcio Cataldi ${ }^{3}$ \\ ${ }^{1}$ Analista de Geomarketing - TIM Celular S.A., RJ \\ leonardopreza@gmail.com \\ ${ }^{2}$ Departamento de Análise Geoambiental - Universidade Federal Fluminense, RJ \\ monicasenna@id.uff.br \\ ${ }^{3}$ Departamento de Engenharia Agrícola e Meio Ambiente - Universidade Federal Fluminense, RJ \\ marcio.cataldi@gmail.com
}

\begin{abstract}
Resumo
Eventos atmosféricos extremos ganham cada vez mais destaque nas discussões acadêmicas e em reportagens vinculadas a mídia, como a Zona de Convergência do Atlântico Sul (ZCAS), que é um dos principais sistemas meteorológicos que influenciam o regime pluviométrico do Brasil durante os meses de verão. Esta relevância faz do fenômeno um processo importante a ser mapeado e compreendido. Este trabalho busca entender como o modelo acoplado atmosfera-biosfera CCM3-IBIS representa a climatologia da formação e ocorrência da ZCAS e seus padrões de comportamento em diferentes níveis atmosféricos. Foi realizada uma simulação controle, por um período de 50 anos, e foram elaborados mapas médios mensais das seguintes variáveis atmosféricas: Vento (Escoamento), Divergência, Umidade Específica, Omega, Radiação de Onda Longa Emitida, Nebulosidade, e Precipitação. O modelo CCM3-IBIS conseguiu representar de maneira satisfatória as características da ZCAS, onde os campos de convergência em baixos níveis, velocidade Omega negativa em níveis médios, divergência em altos níveis, e altos valores de umidade específica em superfície, se mostram em fase, ou seja, concordando espacialmente entre si, corroborando a presença de nebulosidade convectiva, associada a baixos valores de radiação de onda longa emitida e altos valores de precipitação, com orientação NW-SE estendendo-se da região convectiva da Amazônia à região sudeste da América do Sul, e seguindo para o Oceano Atlântico extratropical.
\end{abstract}

Palavras-chave: ZCAS. Precipitação. Climatologia. Modelagem. CCM3-IBIS

\begin{abstract}
Extreme weather events are becoming more prominent in academic discussions and media articles, such as South Atlantic Convergence Zone (SACZ), which is a major weather system that influences rainfall in Brazil during the summer months. The relevance of the phenomenon makes it an important process to be mapped and understood. This work seeks to understand how the biosphere-atmosphere coupled model CCM3-IBIS represents the climatology of the SACZ occurrence and formation, and its behavior patterns at different atmospheric levels. The control simulation was run for a period of 50 years, and average monthly maps of the following environmental variables were elaborated: Wind, Divergence, Specific Humidity, Omega, Outgoing Longwave Radiation, Cloudiness and Precipitation. The CCM3-IBIS model represented satisfactorily the characteristics of the $S A C Z$, where areas of low level convergence, negative speed Omega in average levels, divergence at high levels, and high values of specific surface moisture appear on phase, i.e., spatially agreeing with each other, confirming the presence of convective clouds associated with low values of outgoing longwave radiation and high precipitation rates, oriented NW-SE from the convective region of the Amazon to southeastern South America, reaching the extratropical Atlantic Ocean.
\end{abstract}




\section{Introdução}

Assuntos relacionados ao tempo e ao clima ganham cada vez mais destaque nas discussões acadêmicas e em reportagens vinculadas a mídia, já que a consciência ambiental da população vem sendo cada vez mais expandida devido aos efeitos, muitas vezes catastróficos, de alguns fenômenos meteorológicos sobre a qualidade de vida e a segurança humana. Este fator faz com que eventos como a Zona de Convergência do Atlântico Sul (ZCAS) deixem os livros técnicos e sejam cada vez mais abordados em reportagens e telejornais.

Grande parte da população associa o fenômeno da ZCAS com as chuvas intensas que atingiram as cidades da região serrana do estado do Rio de Janeiro em 2011, provocando o falecimento de mais de 900 pessoas devido a deslizamentos e enchentes. Não obstante, grandes acumulados de precipitação no ano de 2010 provocaram mais de 200 mortes na Região Metropolitana do Rio de Janeiro, sendo 167 mortes em Niterói (Moura et al., 2013).

A ZCAS pode ser compreendida como uma faixa de nebulosidade convectiva que se estende do noroeste ao sudeste da América do Sul, podendo chegar ao Oceano Atlântico Subtropical (Streten, 1973; Kodama, 1992), atua principalmente nos meses de dezembro e janeiro, quando as chuvas acontecem com maior frequência e intensidade, pois durante o verão o escoamento convergente de umidade na baixa troposfera se intensifica. Já no final de março, toda essa atividade convectiva se enfraquece.

Uma das características básicas da ZCAS é persistência da precipitação associada ao sistema com duração mínima de 4 dias chegando a mais de 10 dias. Uma duração inferior a 4 dias faz o evento ser classificado em ZCOU - Zona de Convergência de Umidade (Pallotta e Nakazato, 2010).

A ZCAS ocorre repetidamente em praticamente todos os verões, porém podem existir variações na intensidade de chuva e na circulação atmosférica. São estas variações que ocasionam eventos estocásticos, tendo como resultado alagamentos, deslizamentos, dentre outras fatalidades. Por outro lado, qualquer redução ou ausência das chuvas pode gerar um grande período de seca e má distribuição da mesma, impactando diretamente o sistema de abastecimento hídrico e a geração de energia elétrica do Brasil, já que os maiores reservatórios de armazenamento para a geração de energia e captação de água para consumo humano do país estão situados na área de atuação da ZCAS.

Compreender o comportamento e os padrões de distribuição de chuvas durante a ocorrência de ZCAS é fundamental, dado que o evento impacta o regime hídrico da região mais populosa do país, a região sudeste, que acomoda 80,35 milhões de habitantes (IBGE/CENSO, 2010). Todo este montante populacional justifica o quão importante é compreender a variabilidade de chuvas causada pela ZCAS e sua subordinação ao sistema de abastecimento de água da região.
Atrelado ao caráter hídrico de abastecimento está o uso na atividade agrícola e pecuária, visto que as mesmas necessitam de padronização no regime pluvial. A mais impactada, de certa forma, pelo prolongado período de seca são as plantações. Desta forma surge a seguinte dialética: a falta de precipitação prejudica o abastecimento de água e sua utilização nas atividades agropecuárias, no entanto, seu excesso provoca desastres naturais. As causas relacionadas à segunda consequência muitas vezes são fruto do mau planejamento urbano por parte das autoridades, assim como atitudes da população, que por falta de oportunidade acaba ocupando locais de risco.

Segundo Carvalho et al. (2004) quando a ZCAS possui atividade convectiva sobre o Oceano Atlântico, aumentam os casos extremos de precipitação sobre o Planalto Paulista e a Serra da Mantiqueira. Todavia, quando a atividade convectiva aumenta sobre o continente e reduz sobre o oceano, os eventos extremos ocorrem em praticamente todas as regiões do Brasil, porém diminuem significativamente sobre a Serra da Mantiqueira. No estudo de Carvalho et al. (2004) foi comprovado que a atividade convectiva associada a ZCAS sobre o continente aumenta em $95 \%$ o percentil da precipitação diária sobre o Centro-Leste e parte do Nordeste do Brasil. Em ZCAS de menor intensidade há redução desse percentil.

Muito se discute sobre a localização e a caracterização da ZCAS, contudo, através de modelos climáticos e estudos numéricos é possível mensurar e compreender o evento climático. Figueroa et al. (1995) demonstraram através de simulações numéricas, que é graças ao grande aquecimento adiabático na Amazônia que ocorre a formação da ZCAS, e não devido a localização dos Andes apenas.

A variação temporal da ZCAS é um fator que merece estudo e atenção, pois a forte atividade convectiva no oeste e sudoeste da Amazônia provoca o surgimento e acumulação de frentes frias sobre o sul do Brasil e a Argentina, o que reflete a capacidade de abrangência do fenômeno de ZCAS e sua influência no âmbito continental (Cavalcanti et al., 2009).

Dado o exposto, este trabalho teve como objetivo investigar como o modelo acoplado atmosfera-biosfera CCM3-IBIS representa a climatologia da formação e ocorrência da ZCAS e seus padrões de comportamento em diferentes níveis, validando se a representação estimada pelo modelo é aderente à realidade. Este é um tema relevante, dada a grande influência da ZCAS na precipitação e ocorrência de eventos extremos nos centros urbanos das Regiões Sul e Sudeste do Brasil. Além disso, a representação satisfatória da ocorrência da ZCAS pode favorecer a previsão de sua variabilidade e de seus impactos na atividade humana considerando cenários futuros do clima.

\section{Materiais e Métodos}




\subsection{Modelo Utilizado}

Durante este estudo foi utilizado o modelo de circulação geral da atmosfera CCM3 acoplado ao modelo integrado de simulação da biosfera IBIS (Delire et al., 2003). O CCM3 (Community Climate Model) é um modelo de circulação geral da atmosfera, desenvolvido pela divisão de clima e dinâmica global do NCAR (National Center for Atmospheric Research) (Kiehl et al., 1998).

A pesquisa utilizou a versão 3.6.16, com resolução T42 $\left(\sim 2,8^{\circ} \times 2,8^{\circ}\right)$. Além disso, foram considerados 18 níveis na vertical, em um sistema de coordenadas híbrido sigma-pressão (sigma próximo à superfície, pressão no topo da atmosfera). O CCM3 tem sido significativamente testado pela comunidade científica internacional, sendo um dos modelos climáticos mais utilizados em todo o mundo.

O modelo biosférico IBIS (Integrated Biosphere Simulator) compõe uma nova geração de modelos da biosfera global, denominado como modelo dinâmico da vegetação global. O mesmo considera as mudanças ocorridas na composição e estrutura da vegetação em resposta às condições ambientais (Foley et al., 1996).

O módulo da superfície terrestre do IBIS simula trocas de energia, água, carbono e momentum no sistema de interação solo-vegetação-atmosfera. O IBIS apresenta duas camadas de vegetação simuladas (árvores e gramíneas), oito diferentes tipos de solo e opera em diferentes intervalos de integração, que poderem variar entre 60 minutos a 1 ano. $\mathrm{O}$ mesmo ainda permite o acoplamento de processo ecológicos, biofísicos e fisiológicos que ocorrem em escalas de tempo diferenciadas (Kucharik et al., 2000). Foi utilizada a versão 2.6.4 calibrada para quatro sítios de floresta tropical amazônica (Imbuzeiro, 2005).

A função básica do modelo acoplado CCM3-IBIS é simular as interações biofísicas de curto prazo entre a superfície e a atmosfera através das trocas de energia, água, carbono e momentum. Subsequentemente, o modelo simula as retroalimentações de longo prazo entre o ecossistema e o clima, gerando modificações na cobertura vegetal e nos reservatórios de carbono.

Com essa modelagem é possível considerar as retroalimentações biogeofísicas da cobertura vegetal no clima regional, por exemplo na representação da ZCAS, que é um fenômeno fortemente impactado pela evapotranspiração amazônica. Nos últimos anos o CCM3-IBIS foi recalibrado usando dados do experimento LBA (Experimento de Grande Escala da Biosfera-Atmosfera na Amazônia) e de sensoriamento remoto. Sendo testado extensivamente em cenários de clima atual e futuro, como é possível observar nos trabalhos de Pereira (2006), Yanagi (2006), Senna et al. (2009), e Senna et al. (2009b).

\subsection{Delineamento Experimental para o CCM- 3-IBIS}

O experimento foi efetuado na resolução T42 $\left(\sim 2,8^{\circ}\right.$ x $\left.2,8^{\circ}\right)$, com vegetação dinâmica e por um período de 50 anos. A simulação realizada para validação do comportamento do modelo foi denominada de Controle. A concentração atmosférica de $\mathrm{CO}_{2}$ foi mantida constante, como o valor de 380 ppmv e a temperatura da superfície do mar foi considerada como a média dos anos 1990. Foi considerada a cobertura de vegetação potencial na América do Sul (vegetação que poderia existir na ausência de intervenção humana) conforme o mapa elaborado por Ramankutty e Foley (1999). A climatologia obtida pelo modelo representa a média mensal de 50 anos de simulação controle.

A partir desta simulação controle foi feita uma climatologia mensal dos comportamentos atmosféricos em diversos níveis de altitudes. Foram escolhidas sete variáveis simuladas, com objetivo de analisar a representação da ZCAS, tais variáveis utilizadas e suas unidades são: Vento (Escoamento) em m/s, Divergência em $\mathrm{s}^{-1}$, Umidade Específica em $\mathrm{kg} / \mathrm{kg}$, Velocidade Omega em $\mathrm{Pa} / \mathrm{s}$, Radiação de Onda Longa Emitida em $\mathrm{W} / \mathrm{m}^{2}$, Nebulosidade em fração, e Precipitação em mm/dia. Entretanto, algumas variáveis foram estipuladas em níveis diferenciados, como por exemplo, o Escoamento e a Divergência Horizontal foram avaliados nos níveis de 200 e 850 hPa, Umidade Específica em 1000 hPa e o Omega em $500 \mathrm{hPa}$. Com a análise dessas variáveis simuladas, diversos fenômenos atmosféricos podem ser identificados.

\section{Resultados e Discussão}

Nesta sessão serão descritos os mapas referentes à climatologia simulada para o mês de janeiro para validar a representação da ZCAS, já que é durante este mês que o fenômeno ocorre, em média, de maneira mais intensa, além de corresponder ao principal mês de caracterização do verão no hemisfério sul.

\subsection{Escoamento Horizontal e Divergência nos Níveis de 850 e $200 \mathrm{hPa}$}

Os mapas de escoamento horizontal $(\mathrm{m} / \mathrm{s})$ apresentam a orientação dos ventos tanto nos níveis de 850 e $200 \mathrm{hPa}$. Já os mapas de divergência $\left(\mathrm{s}^{-1}\right)$ apresentam as linhas de correntes referentes ao escoamento horizontal e as cores ao fundo referem-se a divergência propriamente dita (Figuras 1 e 2). É importante sinalizar que a unidade de medida referente aos níveis de pressão atmosférica é inversamente proporcional ao de altitude, quanto maior o valor da pressão em $\mathrm{hPa}$, mais próxima a faixa está da superfície.

Na Figura 1a referente ao escoamento horizontal em $850 \mathrm{hPa}$ durante o mês de janeiro, é clara a existência do evento atmosférico denominado Jatos de Baixos Níveis da América da Sul (JBNAS), evento que possui como origem a canalização dos alísios que penetram no conti- 


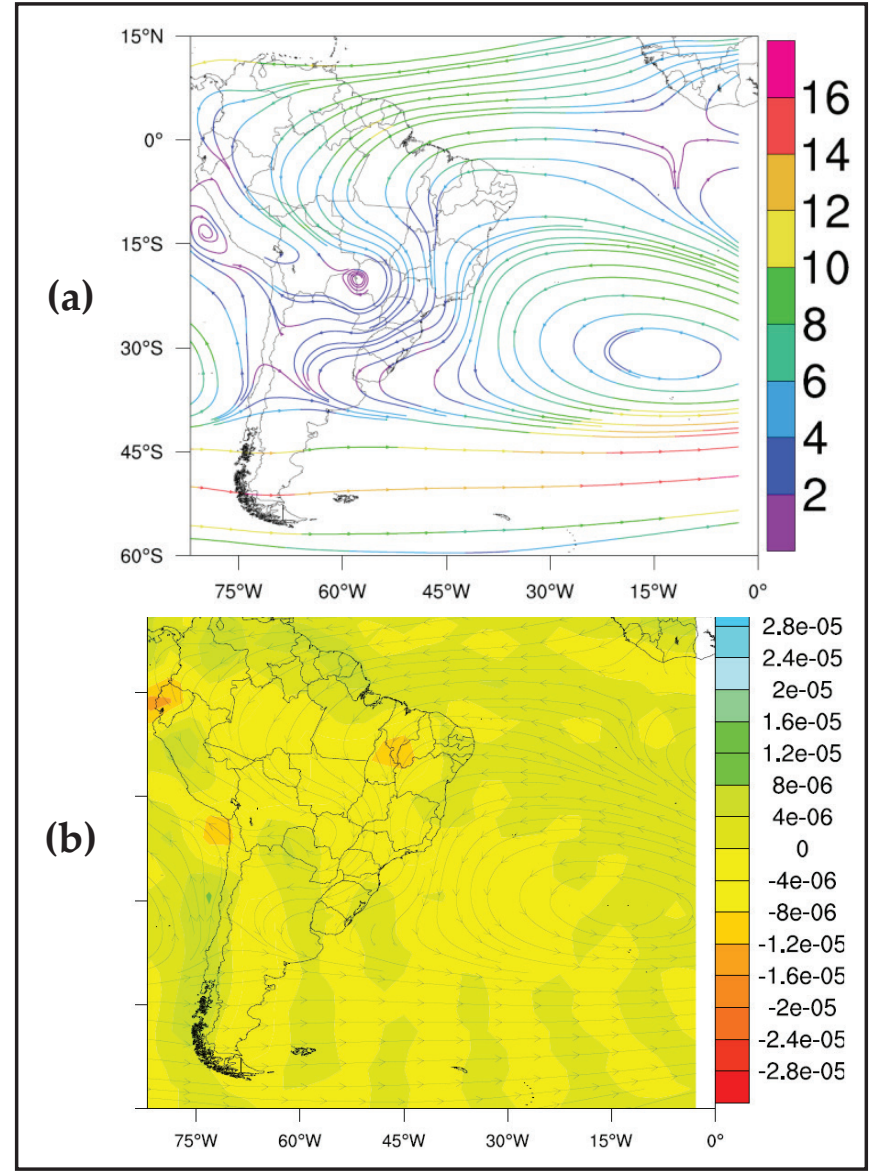

Figura 1 - A figura (a) representa o Escoamento Horizontal (m/s) em $850 \mathrm{hPa}$ e a figura (b) o Escoamento com a Divergência $\left(\mathrm{s}^{-1}\right)$ no mesmo nível $(850 \mathrm{hPa})$, os dois mapas são referentes ao mês de Janeiro

nente sul-americano na região tropical, intensificando o vapor d'água oriundo do Atlântico Equatorial e da Bacia Amazônica em direção ao Atlântico Sudeste (Pallota e Gan, 2011). O JBNAS médio simulado em janeiro corta os estados de Rondônia, Mato Grosso, Mato Grosso do Sul até chegar a Região Sudeste, local do fim do deslocamento (ponto mais a leste do Brasil que o jato alcança).

É notória a predominância do escoamento horizontal no sentido NW-SE do Brasil, o que favorece o transporte de umidade para o Sul e Sudeste do país. Na Figura 1b é possível observar que a divergência é negativa (ou seja, temos uma convergência) no corredor no sentido NW-SE, localização preferencial da ZCAS. Além disso, o giro anticiclônico resultante do Anticiclone Subtropical do Atlântico Sul (ASAS) é bem delimitado, cujo centro situa-se aproximadamente em $30^{\circ} \mathrm{S}$; $15^{\circ} \mathrm{W}$ (Figura 1a).

Os mapas de escoamento horizontal e divergência simulados em altos níveis (200 hPa) são representados na Figura 2. Neste nível, a representação da Alta da Bolívia ocorre de maneira satisfatória (Figura 2a). A Alta da Bolívia é delimitada na forma de vórtice com circulação anti-horária próxima a Bolívia. $\mathrm{O}$ fenômeno da Alta da Bolívia é oriundo da liberação de calor latente, tendo como origem a convecção amazônica e de um ciclone térmico em baixos níveis (Baixa do Chaco).

Os escoamentos em altos níveis são respostas aos processos dinâmicos e termodinâmicos ocorridos em superfície e evidenciam a convergência de umidade em baixos níveis. No processo de ZCAS é marcante os valores divergentes positivos em $200 \mathrm{hPa}$ na orientação principal NW-SE, conforme representado na Figura $2 b$, comprovando que a área delimitada encontra-se em fase com a área de convergência do ar em níveis mais baixos da troposfera (Figura 1b).

Na figura 2a o Cavado do Nordeste é simulado significativamente, com o eixo central sobre a área tropical oeste do Oceano Atlântico. É importante diferenciar que a Alta da Bolívia nada mais é que a resposta atmosférica a elevadas temperaturas na média troposfera do Brasil Central. Já o Cavado do Nordeste interage na baixa troposfera, impedindo a formação de nuvens e de chuva próximas ao seu centro. Contudo, próximo as suas bordas podem ocorrer precipitações intensas (Santos e Buchmann, 2010).

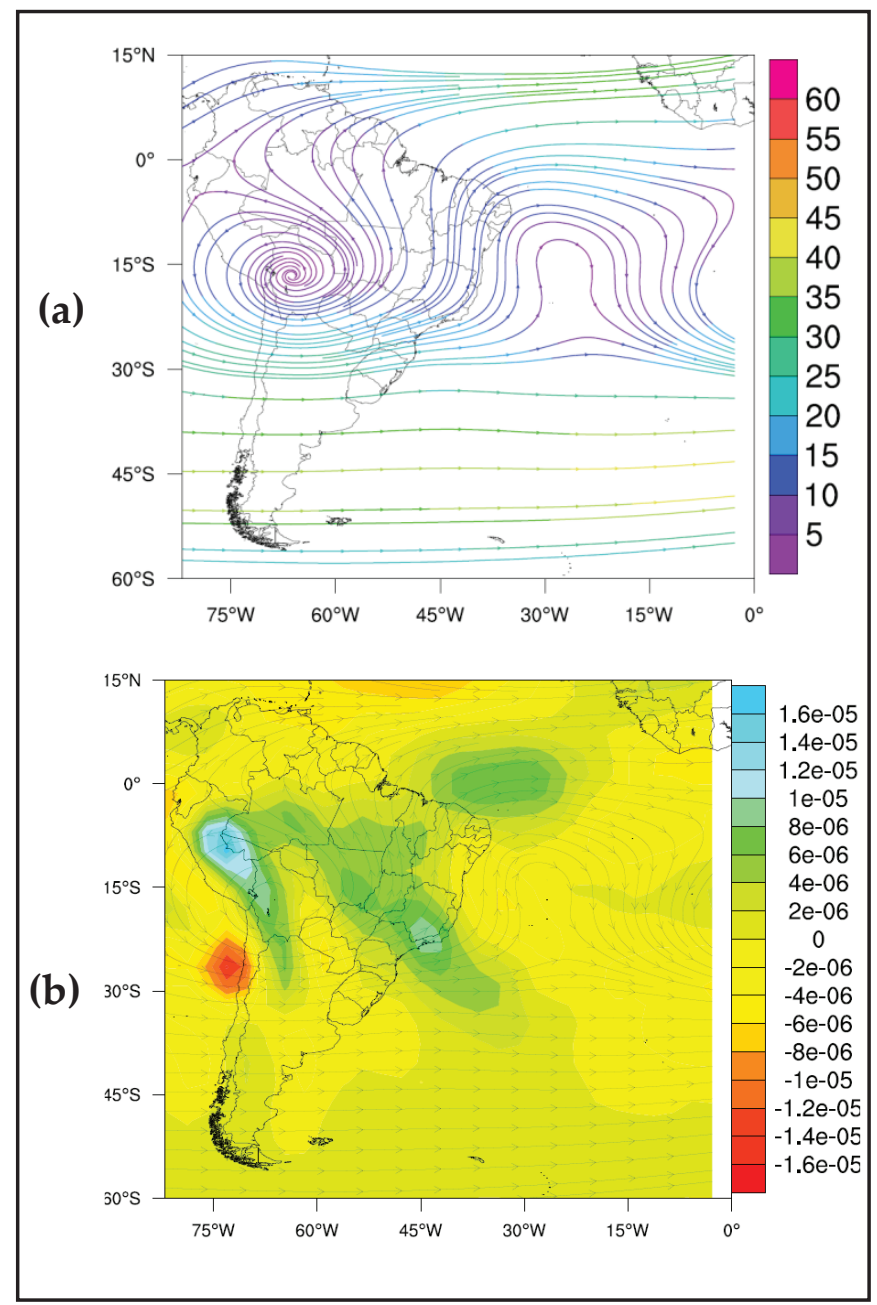

Figura 2 - A figura (a) representa o Escoamento Horizontal (m/s) em 200 hPa e a figura (b) o Escoamento com a Divergência $\left(\mathrm{s}^{-1}\right)$ no mesmo nível, os dois mapas são referentes ao mês de Janeiro 


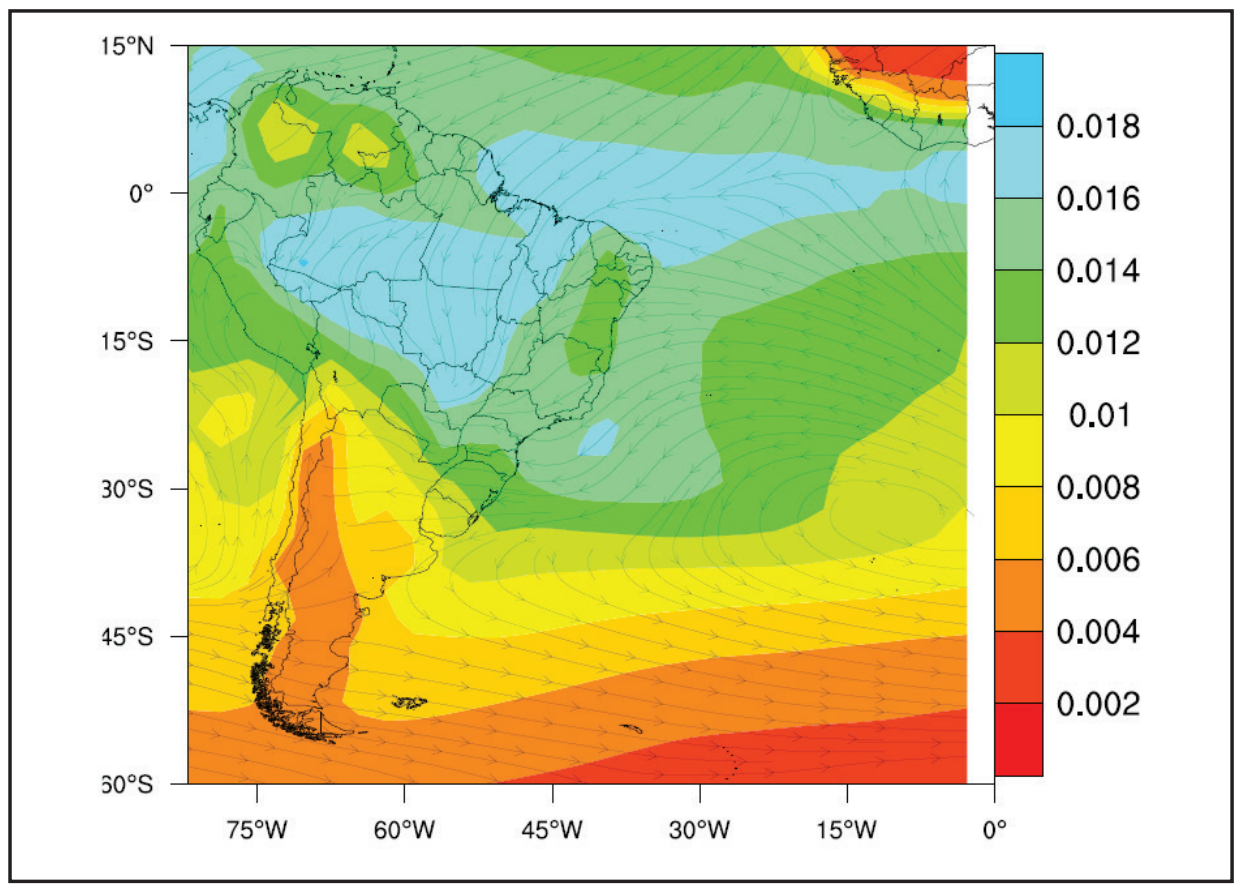

Figura 3 - Representação do Escoamento e da Umidade Específica (kg/kg) em 1000 hPa durante o mês de Janeiro

\subsection{Umidade Específica no Nível de $1000 \mathrm{hPa}$}

Para análise de umidade específica $(\mathrm{Kg} / \mathrm{Kg})$ foi utilizada a camada de baixo nível em $1000 \mathrm{hPa}$, já que esta variável apresenta maior representatividade neste nível atmosférico. É válido ressaltar que os resultados obtidos na simulação controle refletem a característica de ZCAS (Figura 3). Neste é possível observar que os maiores valores ocorrem sobre a Amazônia caminhando até a Região Sudeste. Isto ocorre devido a influência direta da evapotranspiração oriunda da floresta tropical amazônica e o JBNAS.

Toda esta umidade provoca retroalimentação da atividade convectiva, e os altos valores de umidade específica possuem orientação NW-SE, sendo os valores acima de $0,016 \mathrm{~kg} / \mathrm{kg}$ retidos até a Região Centro-Oeste e em uma pequena área oceânica próxima ao litoral do Rio de Janeiro. Com isso, pode-se concluir que o modelo representa um evento de ZCAS, já que os maiores valores de umidade encontram-se em fase com a convergência do ar em 850 hPa e divergência em 200 hPa.

A variável umidade é de grande importância na análise do potencial de uma ZCAS, quando ocorre escoamento horizontal de umidade é comum a variação na organização espacial, na intensidade das precipitações e na circulação atmosférica. Esta variável é na maior parte das vezes a responsável pela manifestação de eventos severos, inundações e deslizamentos de terra (Justi da Silva e al, 2004).

\subsection{Omega no Nível de 500 hPa}

A variável Omega $(\mathrm{Pa} / \mathrm{s})$ foi analisada em níveis médios (500 hPa), e o seu comportamento durante o mês de Janeiro pode ser visto na Figura 4. Através do mapa é possível observar a grande faixa de valores negativos de Omega na orientação NW-SE do Brasil, o que significa uma velocidade vertical ascendente do vento na mesma região onde ocorre convergência em $850 \mathrm{hPa}$, divergência em $200 \mathrm{hPa}$ e altos valores de umidade específica em $1000 \mathrm{hPa}$. Esta característica atmosférica contribui para a presença de nebulosidade convectiva na orientação NW-SE. Este padrão encontrado na simulação controle em janeiro é um exemplo clássico de formação de um evento da ZCAS de grande magnitude.

Além disso, o Cavado simulado a leste da Cordilheira dos Andes com orientação NW-SE suporta a existência de nebulosidade e da precipitação, pois usualmente a leste do cavado localiza-se uma região com intensa vorticidade negativa, gerando convergência do ar em superfície (Pallota e Gan, 2011).

\subsection{Radiação de Onda Longa Emitida, Nebulo- sidade e Precipitação}

Devido às suas inter-relações, as variáveis Radiação de Onda Longa Emitida (ROL) (W/m²), Nebulosidade (fração) e Precipitação ( $\mathrm{mm} /$ dia) serão analisadas conjuntamente. A ROL simulada (Figura 5) possui os menores valores na faixa NW-SE, local de ocorrência da ZCAS. Quando os valores de ROL apresentam números absolutos reduzidos, é uma indicação de que a radiação de onda longa está sendo emitida pelo topo das nuvens, usualmente muito mais frios que a superfície do solo. Quanto maior for a temperatura de um corpo, mais radiação ele emitirá (e vice-versa), conforme a Lei de Stefan-Boltzmann. Além disso, a variável ROL é 


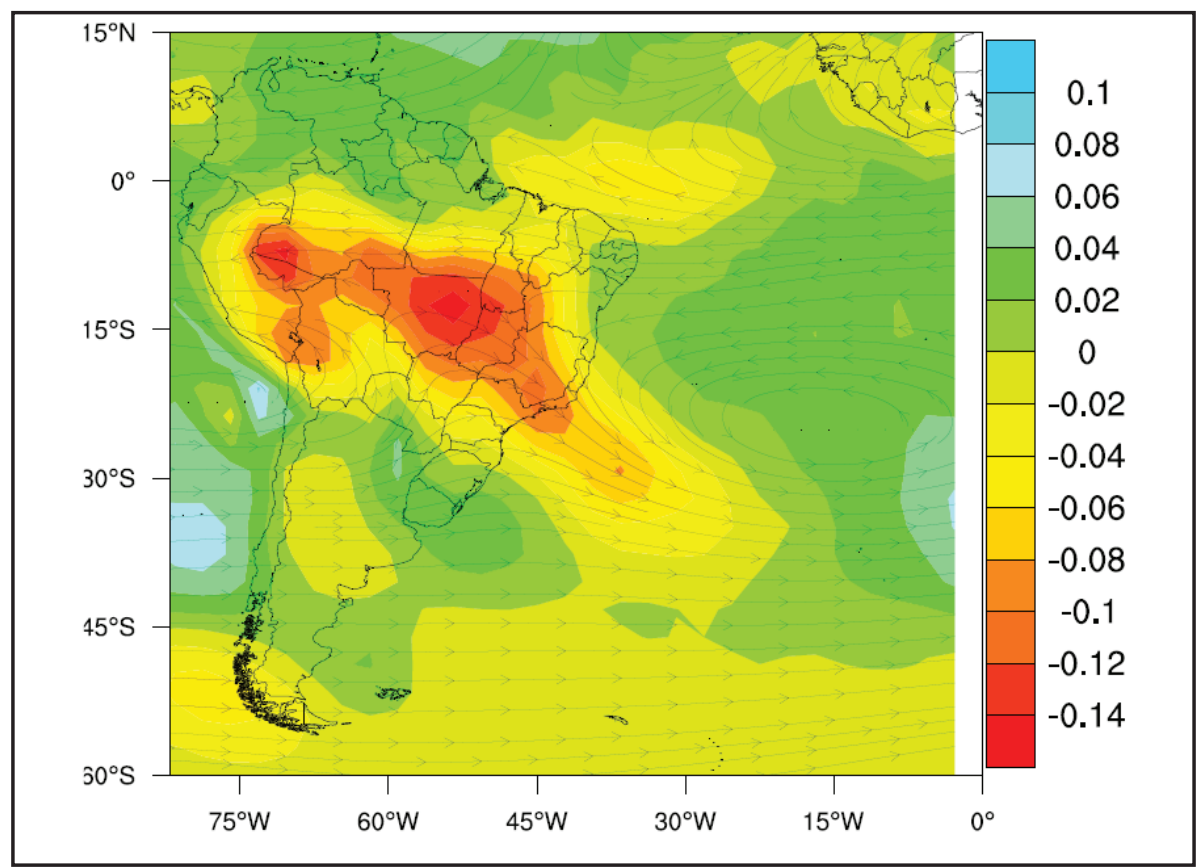

Figura 4 - Representação do Escoamento e de Omega (Pa/s) no nível de 500 hPa durante o mês de Janeiro

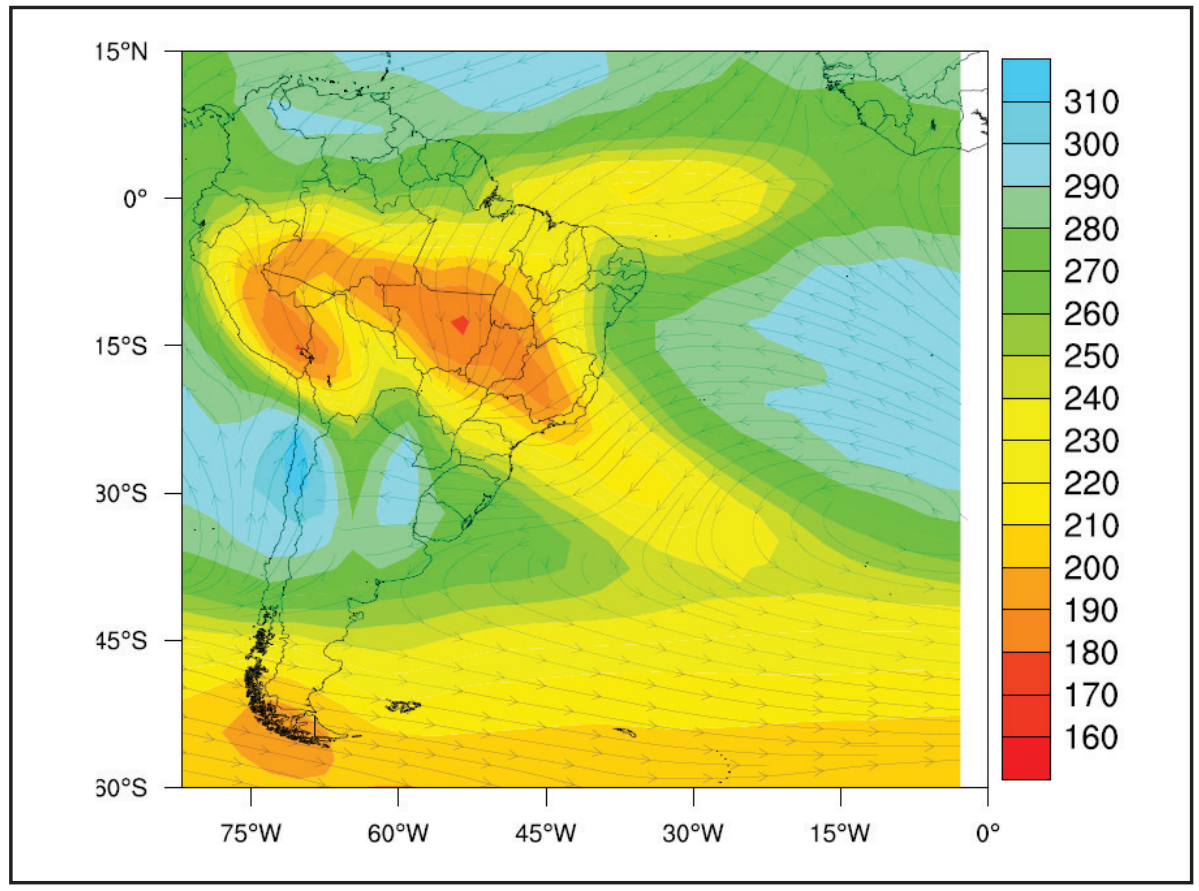

Figura 5 - Representação do Escoamento em 1000 hPa e da Radiação de Onda Longa (ROL) emitida pela superfície ou pela nebulosidade, referente ao mês de Janeiro, em $\mathrm{W} / \mathrm{m}^{2}$

intrinsecamente relacionada à precipitação.

Para analisar a representação de nebulosidade referente ao mês de janeiro da simulação controle é necessário considerar que todas as regiões que apresentarem coloração tendendo a tons mais frios na Figura 6, possuem uma extensa área coberta por nebulosidade. Por exemplo, caso alguma região seja representada pela cor azul correspondente a 0,9 na escala, significa que $90 \%$ do céu está coberto por nebulosidade.

Ao observar a Figura 6 é notório como a nebulosidade foi simulada adequadamente, similar com a observada durante um evento da ZCAS. Destaca-se a extensa faixa de nebulosidade no sentido NW-SE, ultrapassando os limites continentais, desde o sul da região Amazônica, chegando ao Oceano Atlântico. 


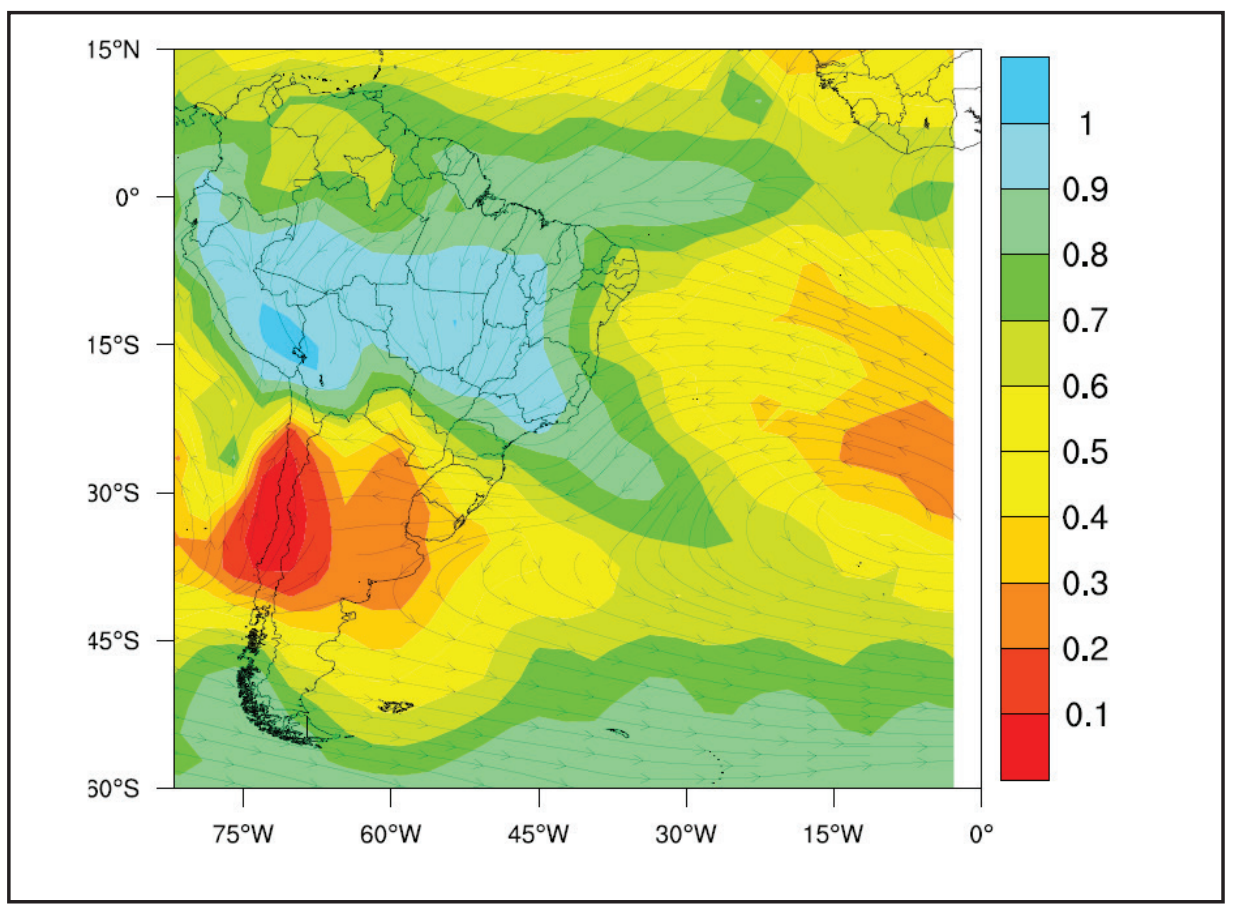

Figura 6 - Representação do Escoamento em 1000 hPa e da Nebulosidade Total (representada por fração) referente ao mês de Janeiro

O mapa da precipitação total simulada em mm/dia (Figura 7) apenas confirma as informações anteriores de ROL e Nebulosidade. O corredor com as maiores precipitações continua representado no sentido NW-SE, onde é possível observar os altos índices de precipitação nas regiões Norte, Sudeste e Centro-Oeste. Através do mapa nota-se a relevância da ZCAS no regime hídrico no
Centro-Oeste, além, é claro, da compreensão do motivo do fenômeno ser tão estudado pelos grandes produtores agrícolas do Brasil (Figura 7). Além da própria relevância que a precipitação possui para os grandes centros urbanos, também devemos considerar a importância desta precipitação para o abastecimento das usinas hidrelétricas que irão fornecer energia.

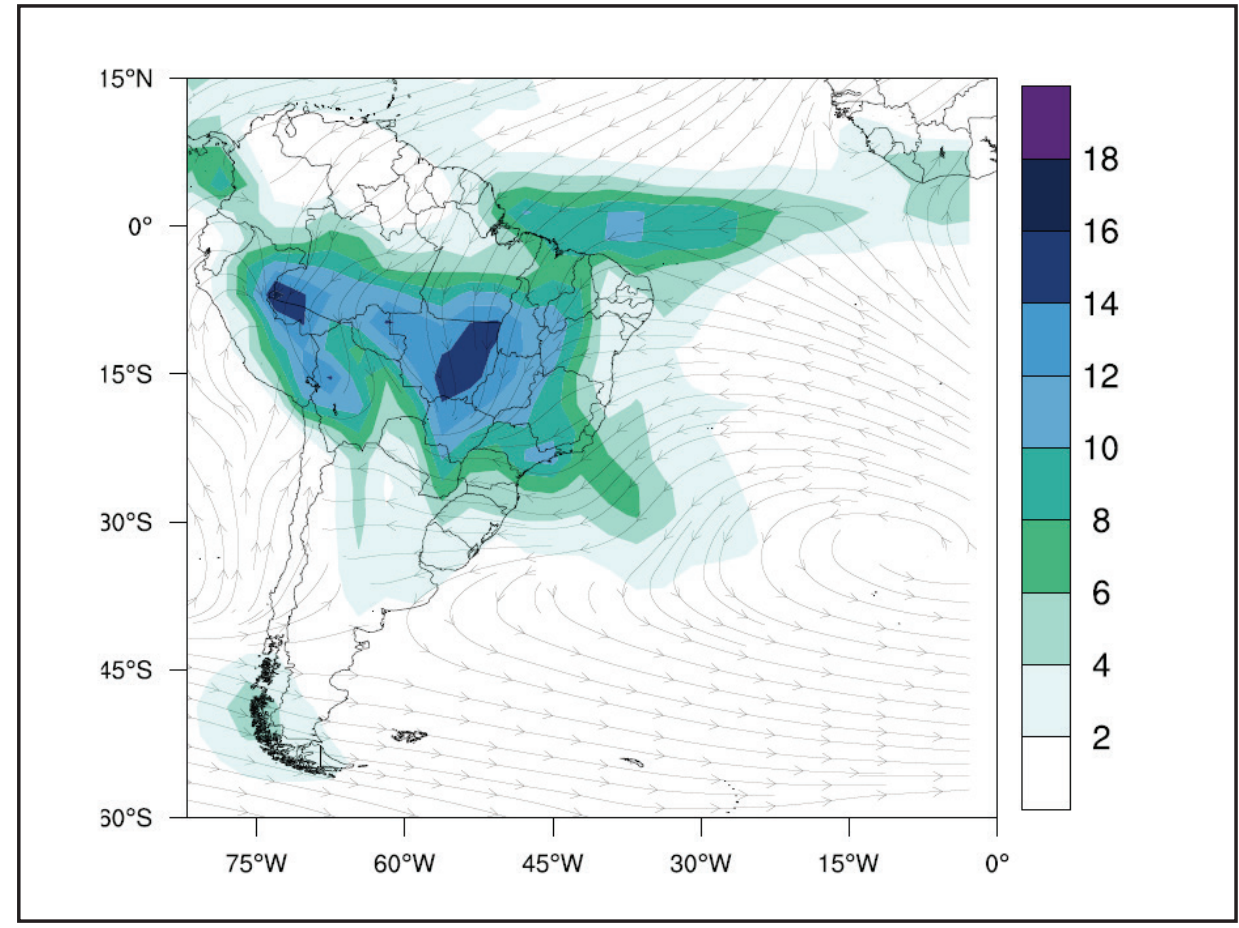

Figura 7 - Representação do Escoamento em 1000 hPa e da Precipitação Total (mm/dia) referente ao mês de Janeiro 


\section{Conclusão}

O principal objetivo do presente trabalho foi investigar como o modelo acoplado atmosfera-biosfera CCM3IBIS representa a climatologia das características atmosféricas da Zona de Convergência do Atlântico Sul (ZCAS). Com essa finalidade, foi realizada uma simulação controle, por um período de 50 anos, e foram elaborados mapas médios mensais das seguintes variáveis atmosféricas: Vento (Escoamento), Divergência, Umidade Específica, Omega, Radiação de Onda Longa Emitida, Nebulosidade, e Precipitação.

Após a análise da climatologia simulada para o mês de janeiro (mês que a ZCAS atua de forma mais intensa), conclui-se que o modelo conseguiu representar os padrões de grande escala da atmosfera necessários à formação e manutenção de um caso de ZCAS clássica, onde os campos de convergência em baixos níveis, velocidade Omega negativa em níveis médios, divergência em altos níveis, e altos valores de umidade específica em superfície, se mostram em fase, ou seja, concordando espacialmente entre si, corroborando a presença de nebulosidade convectiva, associada a baixos valores de ROL emitida e altos valores de precipitação, com orientação NW-SE estendendo-se da região convectiva da Amazônia à região sudeste da América do Sul, e seguindo para o Oceano Atlântico extratropical.

Finalmente, destaca-se que a representação adequada das características da ZCAS é altamente desejável para um modelo climático acoplado, como o CCM3-IBIS, pois permite a sua utilização como ferramenta para o estudo de cenários futuros do clima e seus impactos na ZCAS e consequentemente na precipitação sobre todo o país.

\section{Agradecimentos}

Os autores gostariam de agradecer à FAPERJ pelo apoio financeiro.

\section{Referências}

CARVALHO, L. M. V.; JONES, C.; LIEBMANN, B. The South Atlantic Convergence Zone: Intensity, form, persistence, and relationships with intraseasonal to interannual activity and extreme rainfall. Journal of Climate, v.17, p. 88-108, 2004.

CAVALCANTI, I. F. A. C.; FERREIRA, N. J.; SILVA, M. G. A. J.; DIAS, M. A. F. S. Tempo e Clima no Brasil, São Paulo: Oficina de Texto, 2009

DELIRE, C.; FOLEY, J. A.; THOMPSON, S. Evaluating the carbon cycle of a coupled atmosphere-biosphere model. Global Biogeochemical Cycles, 17 (1): 1012, 2003.
FIGUEROA, S. N.; SATYAMURTY, P.; SILVA DIAS, P. L. Simulations of the summer circulation over the South American region with an Eta coordinate model. J. Atmos. Sci., v.52, p. 1573-1584, 1995.

FOLEY, J. A.; PRENTICE, I. C.; RAMANKUTTY, N.; LEVIS, S.; POLLARD, D.; SITCH, S.; HAXELTINE, A. An integrated biosphere model of land surface processes, terrestrial carbon balance, and vegetation dynamics. Global Biogeochemical Cycles, 10: 603-628, 1996.

IBGE/CENSO. http://censo2010.ibge.gov.br, 2010.

IMBUZEIRO, H. M. A. Calibração do modelo IBIS na Floresta Amazônica usando múltiplos sítios. Dissertação de mestrado, Universidade Federal de Viçosa, 67 p., 2005

JUSTI DA SILVA, M. G. A.; SANTOS, I. A.; COELHO, D. G.; SILVA, A. S. Estudo de eventos meteorológicos extremos na Costa Sul Fluminense através de simulações numéricas. XIII Congresso Brasileiro de Meteorologia, 2004

KIEHL, J. T.; HACK, J. J.; BONAN, G. B.; BOVILLE, B. A.; WILLIAMSON, D. L.; RASCH, P. J. The National Center for Atmospheric Research Community Climate Model: CCM3. Journal of Climate, 11 (6): 1131-1149, 1998.

KODAMA, Y. Large-Scale Common Features of Subtropical Precipitation Zones (the Baiu Frontal Zone, the SPCZ, and the SACZ). Part I: Characteristics of Subtropical Frontal Zones. J. Meteor. Soc. Japan, v.70, p.813-836, 1992.

KUCHARIK, C. J.; FOLEY, J. A.; DELIRE, C.; FISHER, V. A.; COE, M. T.; LENTERS, J. D.; YOUNG-MOLLING, C.; RAMANKUTTY, N. Testing the performance of a dynamic global ecosystem model: water balance, carbon balance, and vegetation structure. Global Biogeochemical Cycles, 14: 795-825, 2000.

MOURA, C. R. W.; ESCOBAR, G. C. J.; ANDRADE, K. M. Padrões de circulação em superfície e altitude associados a eventos de chuva intensa na Região Metropolitana do Rio de Janeiro. Revista Brasileira de Meteorologia, v. 28, n. 3, p. 267-280, 2013.

PALLOTTA, M.; NAKAZATO, R. Y. Caracterização de episódios de Zona de Convergência do Atlântico Sul (ZCAS) e Zona de Convergência de umidade (ZCOU) em janeiro e fevereiro de 2010. XVI Congresso Brasileiro de Meteorologia, 2010.

PALLOTA, M.; GAN, M. A. Caracterização do Mês de Janeiro de 2010 em Relação a Não Ocorrência de ZCAS: Uma Comparação com a Climatologia. IV Simpósio Internacional de Climatologia, 2011. 
PEREIRA, M. P. S. Interação entre ecossistemas terrestres e a atmosfera na Amazônia: conexões biogeofísicas e biogeoquímicas. Dissertação de mestrado, Universidade Federal de Viçosa, 111 p, 2006.

RAMANKUTTY, N.; FOLEY, J. A. Estimating historical changes in global land cover: croplands from 1700 to 1992. Global Biogeochemical Cycles, v. 13, n. 4, p. 997-1027, 1999.

SANTOS, I. A.; BUCHMANN, J. Uma revisão qualitativa enfatizando aspectos climáticos da Amazônia e da região Nordeste do Brasil. Anuário de Instituto de Geociencias - UFRJ, v.33, n.2, 09 - 23, 2010.

SENNA, M. C. A.; COSTA, M. H.; PIRES, G. F. Vegetationatmosphere-soil nutrient feedbacks in the Amazon for different deforestation scenarios. Journal of Geophysical Research, 114 (D04104), doi:10.1029/2008JD010401, 2009.

SENNA, M. C. A.; COSTA, M. H.; PINTO, L. I. C.; IMBUZEIRO, H. M. A.; DINIZ, L. M. F.; PIRES, G. $\mathrm{F}$. Challenges to reproduce vegetation structure and dynamics in Amazonia using a coupled climatebiosphere model. Earth Interactions, 13 (11), doi: 10.1175/2009EI281.1, 2009 b.

STRETEN, N. A. Some characteristic of satellite observed bands of persistent cloudiness over the Southern Hemisphere. Monthly Weather Review, v.101, p. 486495, 1973.

YANAGI, S. N. M. Albedo de uma floresta tropical amazônica: medições de campo, sensoriamento remoto, modelagem, e sua influência no clima regional. Tese de doutorado, Universidade Federal de Viçosa, 128 p, 2006. 